Research Paper

\title{
Bleeding Risk of Warfarin and Direct Oral Anticoagulants in Younger Population: A Historical Cohort Study Using a Japanese Claims Database
}

\author{
Satoshi Yokoyama ${ }^{1}$, Yuki Tanaka ${ }^{1}$, Kazuki Nakagita ${ }^{1,2}$, Kouichi Hosomi ${ }^{1}$, Mitsutaka Takada ${ }^{1}$ \\ 1. Division of Clinical Drug Informatics, Faculty of Pharmacy Kindai University, Japan. \\ 2. Department of Pharmacy, National Cerebral and Cardiovascular Center, Suita, Japan. \\ $\square$ Corresponding author: Satoshi Yokoyama, PhD, Division of Clinical Drug Informatics, Faculty of Pharmacy, Kindai University, 3-4-1 Kowakae, Higashiosaka \\ city, Osaka 577-8502, Japan. Telephone number: +81-6-6721-2332; Fax number: +81-6-6730-1394; E-mail address: yokoyama@phar.kindai.ac.jp \\ (c) Ivyspring International Publisher. This is an open access article distributed under the terms of the Creative Commons Attribution (CC BY-NC) license \\ (https://creativecommons.org/licenses/by-nc/4.0/). See http://ivyspring.com/terms for full terms and conditions.
}

Received: 2018.07.31; Accepted: 2018.09.14; Published: 2018.11.22

\begin{abstract}
A historical cohort analysis of the Japan medical data center (JMDC) claims databases was performed to compare the incidence rates of bleeding events with warfarin (WF) versus direct oral anticoagulant (DOAC) treatment in patients with non-valvular atrial fibrillation. The aim of this study is to clarify the risk factors for bleeding events in younger patients newly treated with WF or DOAC in clinical practice setting. Patients who newly initiated WF or DOAC treatment from April 2012 to March 2015 were selected from the JMDC claims database. A 1:1 propensity score matching analysis was used for new users of WF or DOAC. Kaplan-Meier curves were generated to depict the time to bleeding event (total bleeding events, gastrointestinal hemorrhage, and intracranial hemorrhage) during the follow-up period. Cox proportional regression models were used to estimate the hazard ratios for total bleeding events caused by oral anticoagulants. Overall, 2,046 patients (503 WF and 1,543 DOAC) were included. After applying propensity score matching, Kaplan-Meier analysis of the WF and DOAC groups displayed comparable incidences of total bleeding events, gastrointestinal hemorrhage, and intracranial hemorrhage. Cox proportional hazards modeling showed that the use of WF was not associated with total bleeding events compared with DOAC (hazard ratio: 1.21, 95\% confidence interval: 0.93-1.54, $p=$ 0.15). This historical cohort study using a claims database indicates that the bleeding risk of DOAC was comparable to that of WF in Japanese younger population.
\end{abstract}

Key words: warfarin, direct oral anticoagulant, bleeding event, claims database, historical cohort study

\section{Introduction}

Atrial fibrillation (AF) is one of the most common arrhythmias in clinical practice; it is associated with an increased risk of ischemic stroke and thromboembolic events. AF has become a major public health concern due to the increasing elderly population in Japanese society. Warfarin (WF), a traditional vitamin $\mathrm{K}$ antagonist, has been used to prevent ischemic stroke in patients with AF in clinical practice for decades. Although WF treatment is highly effective for preventing stroke, its significant drawbacks include variable dose requirements as well as a commitment to lifelong, regular, frequent monitoring of the patient's prothrombin time international normalized ratio (PT-INR). WF has a narrow therapeutic index that requires continuous monitoring, and has a number of interactions with numerous dietary factors and medications. These therapeutic limitations consequently lead to failure or discontinuation of treatment.

Recently, 4 direct oral anticoagulants (DOACs) dabigatran, rivaroxaban, apixaban, and edoxaban have been approved for stroke prevention in non-valvular AF in Japan. Several large-scale, randomized, clinical trials demonstrated that DOACs have similar or superior efficacy and safety profiles compared to WF [1-4]. Meta-analysis demonstrated that DOACs have a favorable risk-benefit profile, with significant reductions in stroke, intracranial hemorr- 
hage, and mortality, and with similar rates of major bleeding events compared to WF [5]. Furthermore, DOACs have advantages over WF because their use does not require regular PT-INR monitoring, and they have fewer food and drug interactions. Recent guidelines have recommended the use of DOACs as an alternative to WF in patients with non-valvular AF $[6,7]$.

Adherence to therapy is absolutely essential for patients with non-valvular $\mathrm{AF}$, as the risk of stroke is significantly increased with discontinuation of anticoagulant therapy. Some studies suggested that patients who initiated WF use discontinued its use within a relatively short period after the initiation of therapy [8]. Although the risk of discontinuation of DOACs is lower than that of WF, detailed treatment patterns for DOACs are unknown [9]. DOACs required adequate adherence in order to maintain their therapeutic effect, because of their relatively short half-lives. Balancing the risks of stroke and bleeding is necessary for optimal use of OAC in clinical practice, and modifiable bleeding risk factors must be addressed. However, there are few reports on the association between DOAC treatment patterns, efficacy, and safety among patients with AF in Japan.

Randomized, controlled trials (RCTs) are the gold standard for demonstrating the efficacy of a particular therapy or intervention. However, RCTs are, by design, limited to a subset of patients who are not fully representative of the unselected real-world population. Real-world evidence is considered to better represent routine practice compared with the idealized conditions of an RCT. Data from real-world studies can complement findings from RCTs and, if appropriately designed, can provide valuable information concerning practice patterns and patient characteristics in the actual clinical setting. Real-world evidence studies include non-interventional studies, patient registries, claims database studies, patient surveys, and electronic health record studies. Recently, Yamashita et al. reported that there were no significant differences in stroke/systemic embolism events or major bleeding events for DOAC compared with WF in patients with AF, based on registry data in Japan [10]. The aim of the current study was to examine differences in the risk of bleeding between WF and DOAC using a claims database in Japanese younger population.

\section{Methods}

\section{Data source}

A large claims database constructed by the Japan Medical Data Center Co., Ltd. (JMDC; Tokyo, Japan), using standardized disease classifications and anonymous record linkage [11], was employed in this historical cohort study. This claims database was constructed with monthly claims from medical institutions and pharmacies submitted from January 2005 to April 2016, which included approximately 3.8 million insured persons (approximately 3.2\% of the population), comprised mainly of company employees and their family members. The JMDC database provided information on the beneficiaries, including encrypted personal identifiers, age, sex, International Classification of Diseases 10th revision (ICD-10) procedure and diagnostic codes, as well as the name, dose, and number of days supplied of the prescribed and/or dispensed drugs. All drugs were coded according to the Anatomical Therapeutic Chemical (ATC) classification of the European Pharmaceutical Market Research Association. An encrypted personal identifier was used to link claims data from different hospitals, clinics, and pharmacies. Based on this JMDC database, we defined our study cohort in order to focus on new DOAC and WF users. This study was approved by the Ethics Committees of the Kindai University School of Pharmacy on July 30, 2011.

\section{Patients and study design}

Figure 1 shows the flowchart for patient selection. Patients who newly initiated WF or DOAC treatment from April 2012 to March 2015 were selected from the JMDC claims database. Patients who were 18 years or older at the initiation of WF or DOAC treatment and had a database history $>12$ months before the initiation of WF or DOAC were eligible. In addition, only patients who had at least 1 diagnosis of $\mathrm{AF}$ in the 12 months prior to the initiation of WF or DOAC treatment were enrolled. ICD-10 code I48 was selected to represent a diagnosis of AF. Atrial flutter and valvular atrial fibrillation were excluded from the analysis because these diseases are not approved as indications for DOAC in Japan. Furthermore, patients undergoing valve replacement were also excluded. Patients without data recorded for a follow-up period of at least 6 months, or patients administered WF or DOAC prior to study initiation, were excluded from the analysis.

Fig. S1 shows the study design. The index date was defined as the date of the first prescription of WF or DOAC after AF diagnosis. Patients were followed from the index prescription until the earliest of the following events: outcome of interest during dosing period (Case 1), discontinuation (when the prescription interval exceeded three months, it was judged that the medication was discontinued), switching to another OAC drug (considered censored cases (Case 2)), or the end of the study (Case 3). 


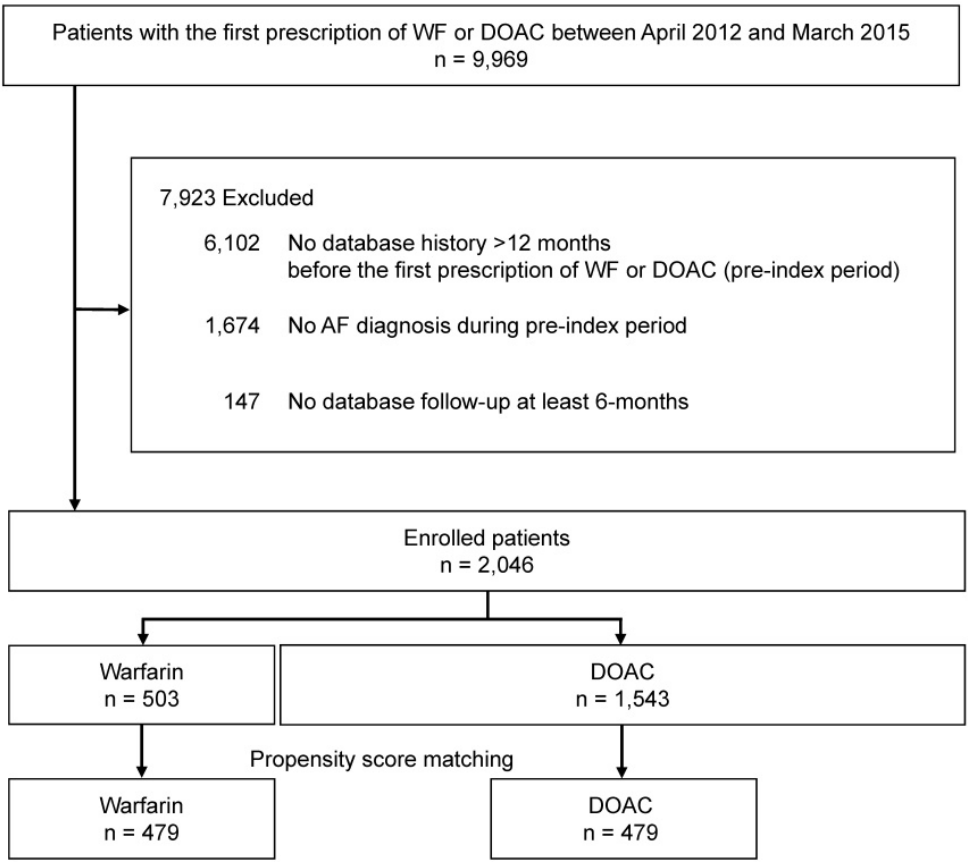

Fig. 1 Patient selection flowchart. AF: atrial fibrillation, WF: warfarin, DOAC: direct oral anticoagulant.

\section{Baseline characteristics}

Patient characteristics were collected in the baseline period. Gender, age, antiplatelet agents (ATC code: B01AC), anti-ulcer agent use (ATC code: A02BA, $\mathrm{H}_{2}$-receptor antagonists; $\mathrm{A} 02 \mathrm{BC}$, proton pump inhibitors (PPI)), heart failure (ICD-10 code: I50), hypertension (ICD-10 code: I10-I13, I15), diabetes mellitus (ICD-10 code: E10-E14), prior stroke or transient ischemic attack (TIA) (ICD10 code: I60-I69, G45), bleeding history, renal dysfunction (ICD-10 code: N17-N19, N28), and gastrointestinal dysfunction (ICD10 code: K25-K29) at baseline were investigated. Additionally, $\mathrm{CHADS}_{2}$ scores (the sum of points for the following conditions: heart failure, hypertension, age $\geq 75$ years, diabetes mellitus, prior stroke or transient ischemic attack (2 points)) [12] were calculated using these baseline characteristics. In order to identify trends in the use of OAC, we also investigated the proportions of WF and DOAC prescribed from 2011 to 2016 in the JMDC claims database.

\section{Study outcome and definitions}

The primary study endpoint was the occurrence of total bleeding events including gastrointestinal hemorrhage, intracranial hemorrhage, and other bleeding events. ICD-10 codes for bleeding events are given in Table S1. Bleeding events were measured during the follow-up period after the initiation of OAC therapy. Secondary endpoints were gastrointestinal hemorrhage and intracranial hemorrhage, defined according to Table S1. Patients were followed from the initiation of anticoagulant therapy to the occurrence of a study outcome of interest, discontinuation of the first anticoagulant drug, or the end of the study period (April 2016).

\section{Statistical analysis}

Categorical variables are summarized as percentages, and continuous variables are summarized as means \pm standard deviations. Between-group comparisons were evaluated using the Student's t-test or chi-square test, as appropriate. To reduce the effect of potential confounding in this observational study, a 1:1 propensity score matching analysis [13] was used to adjust for differences in baseline characteristics (gender, age, antiplatelet agent, $\mathrm{H}_{2}$-receptor antagonists, $\mathrm{PPI}$, renal dysfunction, diabetes mellitus, prior stroke or TIA, hypertension, bleeding event during 12 months before enrollment, heart failure, and gastrointestinal dysfunction) between WF and DOAC cohorts. The propensity score matched pairs were created by matching WF and DOAC groups on the basis of the nearest neighbor pair-matching algorithm with a 0.2 caliper width. The incidences of bleeding outcomes were compared between the WF and DOAC groups after matching. The Kaplan-Meier method was performed to compare the bleeding outcomes of the propensity score matched cohorts, and the groups were compared using the log-rank test. Finally, Cox proportional hazards regression model analyses were used to identify risk factors for bleeding in the whole cohort. All reported $p$ values are 2 -sided, and a $p$ value of $<$ 
0.05 was considered to indicate statistical significance. This analysis was conducted using JMP ${ }^{\circledR}$ version 13.0 (SAS Institute Inc., Cary, NC, USA).

\section{Results}

\section{Study population}

We identified 9,969 patients in the study period who were eligible for inclusion by filling a prescripttion for WF or DOAC. Of these, patients who had no database history during the 12 months before the first WF or DOAC prescription (pre-index period), were < 18 years old, were without at least one AF diagnosis during the pre-index period, or had no database follow-up for at least 6 months were excluded $(\mathrm{n}=$ $7,923)$, leaving 2,046 patients included in the analysis (Fig. 1). These patients were categorized into two groups: the WF group $(n=503)$ and the DOAC group $(\mathrm{n}=1,543)$.

\section{Baseline characteristics}

Table 1 reports baseline characteristics of study patients. Because that population in this study was comprised mainly of company employees and their family members, the range of patients' age included in JMDC data was 0 to 75 years old. The average age was young with $56.6 \pm 9.3$ and $56.8 \pm 9.8$ in WF and DOAC groups, respectively. Gender distribution was balanced between the two groups, and there was no significant difference in mean age between the groups. There were some significant differences in other characteristics. The WF group more frequently used antiplatelet agents (25.1 vs. $10.2 \%), \mathrm{H}_{2}$-receptor antagonists ( 14.9 vs. $7.0 \%$ ), and PPIs (34.0 vs. $24.5 \%$ ) compared with the DOAC group. Significant differences were observed in the prevalence of hypertension ( 67.0 vs. $57.9 \%$ ), renal dysfunction (11.1 vs. $4.5 \%)$, gastrointestinal dysfunction ( 29.4 vs. $23.9 \%$ ), and $\mathrm{CHADS}_{2}$ score (2.31 vs. 2.13) between the WF and DOAC groups. On the index date, the average dose of DOACs: dabigatran, edoxaban, rivaroxaban, and apixaban were $256.8 \pm 48.0,46.4 \pm 18.3,14.0 \pm 2.0$, and $9.6 \pm 1.4 \mathrm{mg}$, respectively. The proportion of patients using each drug of DOACs was following; dabigatran $(36.6 \%)$, edoxaban $(1.4 \%)$, rivaroxaban $(38.1 \%)$, and apixaban $(23.9 \%)$ on index date.

Since DOACs were introduced in 2011 in Japan, there has been an increase in DOAC prescription; the proportion of patients on WF and DOAC was $67.7 \%$ and $32.3 \%$ in 2016, respectively (Fig. 2).

\section{Clinical outcomes}

Kaplan-Meier curves for total bleeding events according to WF and DOAC are shown in Fig. 3a. There was no significant difference in the incidence of total bleeding events between WF and DOAC groups (log-rank test: $p=0.077)$. Kaplan-Meier curves for gastrointestinal hemorrhage events and intracranial hemorrhage events are depicted in Fig. $3 b$ and $3 c$, respectively. A significant difference was observed in the incidence of gastrointestinal hemorrhage between the WF and DOAC groups (log-rank test: $p=0.023$ ), but no significant difference in intracranial hemorrhage was identified (log-rank test: $p=0.738$ ).

Table 1. Characteristics of the study patients

\begin{tabular}{|c|c|c|c|}
\hline Variables & $\begin{array}{l}\text { WF group } \\
(\mathrm{n}=503)\end{array}$ & $\begin{array}{l}\text { DOAC group } \\
(n=1,543)\end{array}$ & $\begin{array}{l}p \\
\text { value }\end{array}$ \\
\hline Age, mean \pm SD & $56.6 \pm 9.3$ & $56.8 \pm 9.8$ & 0.650 \\
\hline Gender male, n (\%) & $419(83.3)$ & $1,306(84.6)$ & 0.473 \\
\hline Antiplatelet agent (ATC code: B01C), n (\%) & $126(25.1)$ & $158(10.2)$ & $<0.001$ \\
\hline $\begin{array}{l}\mathrm{H}_{2} \text {-receptor antagonist (ATC code: A02B1), } \\
n(\%)\end{array}$ & $75(14.9)$ & $108(7.0)$ & $<0.001$ \\
\hline PPI (ATC code: A02B2), n (\%) & $172(34.2)$ & $380(24.6)$ & $<0.001$ \\
\hline Heart failure (ICD10 code: I50), n (\%) & $288(57.3)$ & $828(53.7)$ & 0.160 \\
\hline $\begin{array}{l}\text { Hypertension (ICD10 code: I10-I13, I15), n } \\
(\%)\end{array}$ & $337(67.0)$ & 894 (57.9) & $<0.001$ \\
\hline $\begin{array}{l}\text { Diabetes mellitus (ICD10 code: E10-E14), n } \\
(\%)\end{array}$ & $215(42.7)$ & 708 (45.9) & 0.219 \\
\hline $\begin{array}{l}\text { Prior stroke or TIA (ICD10 code: I60-I69, } \\
\text { G45), n (\%) }\end{array}$ & $161(32.0)$ & $425(27.5)$ & 0.054 \\
\hline $\begin{array}{l}\text { Bleeding event during } 12 \text { months before } \\
\text { enrollment, } \mathrm{n}(\%)\end{array}$ & $37(7.4)$ & $106(6.9)$ & 0.710 \\
\hline $\begin{array}{l}\text { Renal dysfunction (ICD10 code: N17-N19, } \\
\text { N28), n (\%) }\end{array}$ & $56(11.1)$ & $69(4.5)$ & $<0.001$ \\
\hline $\begin{array}{l}\text { Gastrointestinal dysfunction (ICD10 code: } \\
\text { K25-K29), n (\%) }\end{array}$ & $148(29.4)$ & $369(23.9)$ & 0.014 \\
\hline $\mathrm{CHADS}_{2}$ score, mean $\pm \mathrm{SD}$ & $2.31 \pm 1.40$ & $2.13 \pm 1.36$ & 0.010 \\
\hline
\end{tabular}

CHADS 2 score : The sum of points for the following conditions: heart failure, hypertension, age $\geq 75$ years, diabetes mellitus, prior stroke or TIA ( 2 points). WF: warfarin, DOAC: direct oral anticoagulant, SD: standard deviation, PPI: proton pump inhibitor, TIA: transient ischemic attack, ATC: Anatomical Therapeutic Chemical, ICD-10: International Classification of Diseases 10th revision

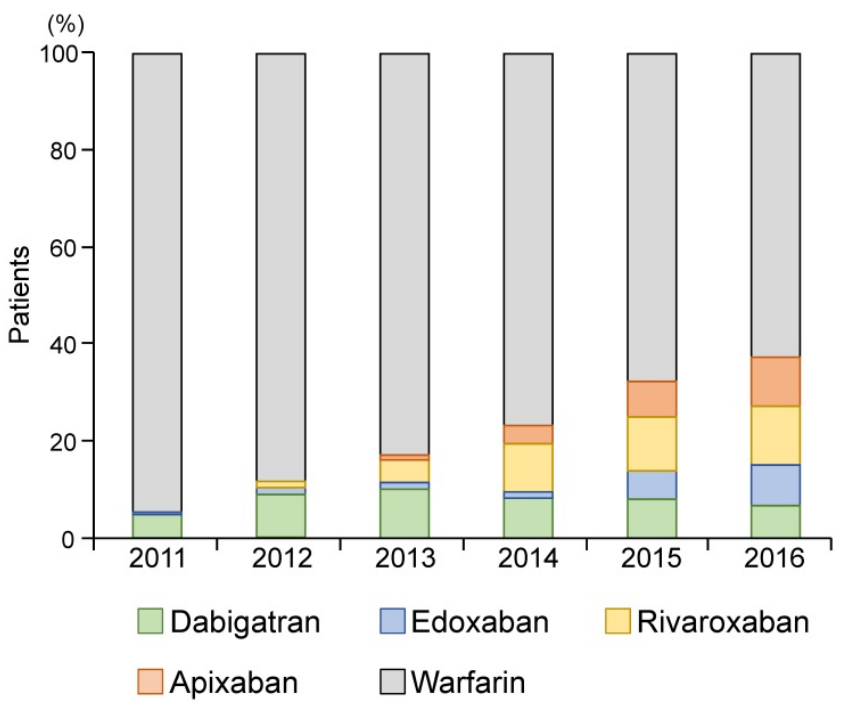

Fig. 2 The proportion of patients on oral anticoagulants from 2011 to 2016. Direct oral anticoagulants are dabigatran, edoxaban, rivaroxaban, and apixaban. DOAC: direct oral anticoagulant.

After applying propensity score matching, 479 patients were matched. The two groups did not differ 
in terms of all clinical variables included in the analysis. Table 2 summarizes all patient data. Kaplan-Meier analysis showed that the WF and DOAC cohorts displayed comparable incidences of total bleeding events (log-rank test: $p=0.582$, Fig. $4 a$ ), gastrointestinal hemorrhage (log-rank test: $p=0.063$, Fig. $4 \mathrm{~b})$, and intracranial hemorrhage (log-rank test: $p$ $=0.482$, Fig. $4 \mathrm{c}$ ).

a)

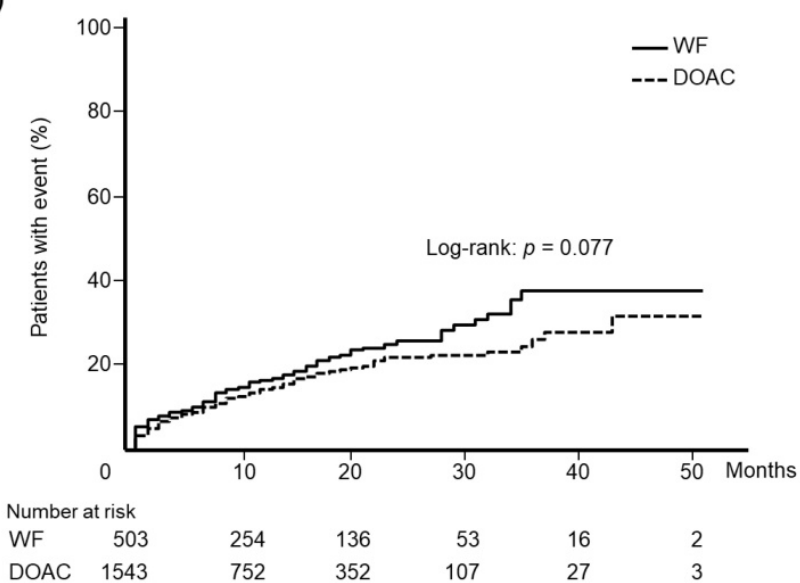

b)

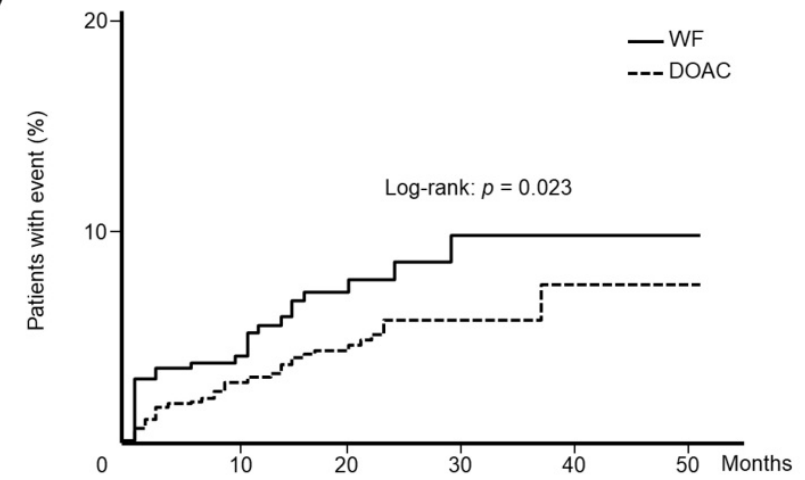

Number at risk

$\begin{array}{lllllll}W F & 503 & 283 & 160 & 68 & 22 & 3\end{array}$

$\begin{array}{lllllll}\text { DOAC } & 1543 & 835 & 419 & 133 & 33 & 3\end{array}$

c)

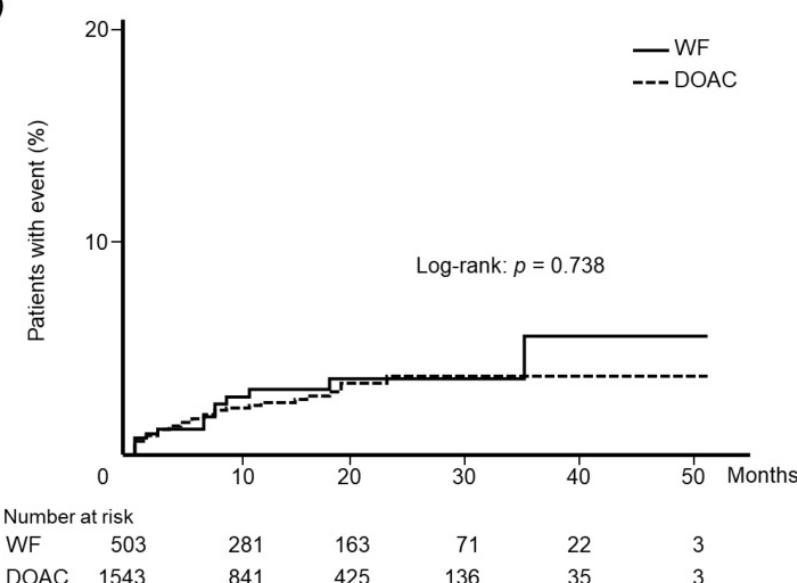

Fig. 3 Kaplan-Meier curves for the incidence of (a) total bleeding events, (b) gastrointestinal hemorrhage, and (c) intracranial hemorrhage among patients newly treated with warfarin or direct oral anticoagulants. WF: warfarin, DOAC: direct oral anticoagulant.
Cox proportional hazards modeling revealed that the use of WF was not associated with total bleeding events compared with DOAC (hazard ratio (HR): $1.21,95 \%$ confidence interval (CI): $0.93-1.54, p=$ 0.148 ). On the other hand, age greater than 60 years old (HR: 1.72, 95\% CI: 1.36-2.17, $p<0.001$ ), prior stroke or TIA (HR: 1.57, 95\%CI: 1.23-1.99, $p<0.001$ ) and gastrointestinal dysfunction (HR: 1.30, 95\% CI: 1.00-1.67, $p=0.048$ ) at baseline were significantly associated with total bleeding events (Table 3).

Table 2. Characteristics of the study patients after propensity score matching

\begin{tabular}{|c|c|c|c|c|}
\hline Variables & $\begin{array}{l}\text { WF group } \\
(\mathrm{n}=479)\end{array}$ & $\begin{array}{l}\text { DOAC } \\
\text { group } \\
(n=479)\end{array}$ & $\begin{array}{l}p \\
\text { value }\end{array}$ & $\begin{array}{l}\text { Standardized } \\
\text { differences }\end{array}$ \\
\hline Age, mean \pm SD & $57.1 \pm 9.5$ & $56.7 \pm 9.2$ & 0.548 & 0.039 \\
\hline Gender male, n (\%) & $398(83.1)$ & $415(86.6)$ & 0.125 & 0.099 \\
\hline $\begin{array}{l}\text { Antiplatelet agent (ATC code: } \\
\text { B01C), n (\%) }\end{array}$ & $105(21.9)$ & $111(23.2)$ & 0.643 & 0.030 \\
\hline $\begin{array}{l}\mathrm{H}_{2} \text {-receptor antagonist (ATC } \\
\text { code: A02B1), } \mathrm{n}(\%)\end{array}$ & $60(12.5)$ & $64(13.4)$ & 0.700 & 0.025 \\
\hline PPI (ATC code: A02B2), n (\%) & $156(32.6)$ & $137(28.6)$ & 0.183 & 0.086 \\
\hline $\begin{array}{l}\text { Heart failure (ICD10 code: I50), } \\
\mathrm{n}(\%)\end{array}$ & $270(56.4)$ & $253(52.8)$ & 0.270 & 0.071 \\
\hline $\begin{array}{l}\text { Hypertension (ICD10 code: } \\
\text { I10-I13, I15), n (\%) }\end{array}$ & $315(65.8)$ & $318(66.4)$ & 0.838 & 0.013 \\
\hline $\begin{array}{l}\text { Diabetes mellitus (ICD10 code: } \\
\text { E10-E14), n (\%) }\end{array}$ & $204(42.6)$ & $202(42.2)$ & 0.896 & 0.008 \\
\hline $\begin{array}{l}\text { Prior stroke or TIA (ICD10 } \\
\text { code: I60-I69, G45), n (\%) }\end{array}$ & $148(30.9)$ & $141(29.4)$ & 0.622 & 0.032 \\
\hline $\begin{array}{l}\text { Bleeding event during } 12 \\
\text { months before enrollment, } \mathrm{n} \\
(\%)\end{array}$ & $35(7.3)$ & $21(4.4)$ & 0.054 & 0.125 \\
\hline $\begin{array}{l}\text { Renal dysfunction (ICD10 } \\
\text { code: N17-N19, N28), n (\%) }\end{array}$ & $44(9.2)$ & $45(9.4)$ & 0.911 & 0.007 \\
\hline $\begin{array}{l}\text { Gastrointestinal dysfunction } \\
\text { (ICD10 code: K25-K29), n (\%) }\end{array}$ & $138(28.8)$ & $136(28.4)$ & 0.886 & 0.009 \\
\hline
\end{tabular}

Table 3. Cox proportional model showing adjusted hazard ratios for the association between total bleeding events and the use of oral anticoagulant

\begin{tabular}{|c|c|c|c|}
\hline Variables & $\begin{array}{l}\text { Hazard } \\
\text { ratio }\end{array}$ & $95 \%$ CI & $\begin{array}{l}p \\
\text { value }\end{array}$ \\
\hline Oral anticoagulant (WF compared with DOAC) & 1.21 & $0.93-1.54$ & 0.148 \\
\hline Age $\geq 60$ years & 1.72 & $1.36-2.17$ & $<0.001$ \\
\hline Gender: male & 0.81 & $0.62-1.09$ & 0.158 \\
\hline Antiplatelet agent (ATC code: B01C), n (\%) & 1.02 & $0.74-1.38$ & 0.916 \\
\hline $\mathrm{H}_{2}$-receptor antagonist (ATC code: A02B1), n (\%) & 1.11 & $0.77-1.58$ & 0.563 \\
\hline PPI (ATC code: A02B2), n (\%) & 1.12 & $0.87-1.43$ & 0.387 \\
\hline Heart failure (ICD10 code: I50), n (\%) & 1.07 & $0.85-1.35$ & 0.560 \\
\hline Hypertension (ICD10 code: I10-I13, I15), n (\%) & 0.96 & $0.75-1.23$ & 0.739 \\
\hline Diabetes mellitus (ICD10 code: E10-E14), n (\%) & 1.04 & $0.83-1.32$ & 0.728 \\
\hline $\begin{array}{l}\text { Prior stroke or TIA (ICD10 code: I60-I69, G45), n } \\
(\%)\end{array}$ & 1.57 & 1.23-1.99 & $<0.001$ \\
\hline $\begin{array}{l}\text { Bleeding event during } 12 \text { months before } \\
\text { enrollment, } \mathrm{n}(\%)\end{array}$ & 0.86 & $0.55-1.28$ & 0.470 \\
\hline $\begin{array}{l}\text { Renal dysfunction (ICD10 code: N17-N19, N28), } \\
\mathrm{n}(\%)\end{array}$ & 1.09 & $0.68-1.66$ & 0.706 \\
\hline $\begin{array}{l}\text { Gastrointestinal dysfunction (ICD10 code: } \\
\text { K25-K29) }\end{array}$ & 1.30 & $1.00-1.67$ & 0.048 \\
\hline
\end{tabular}


a)

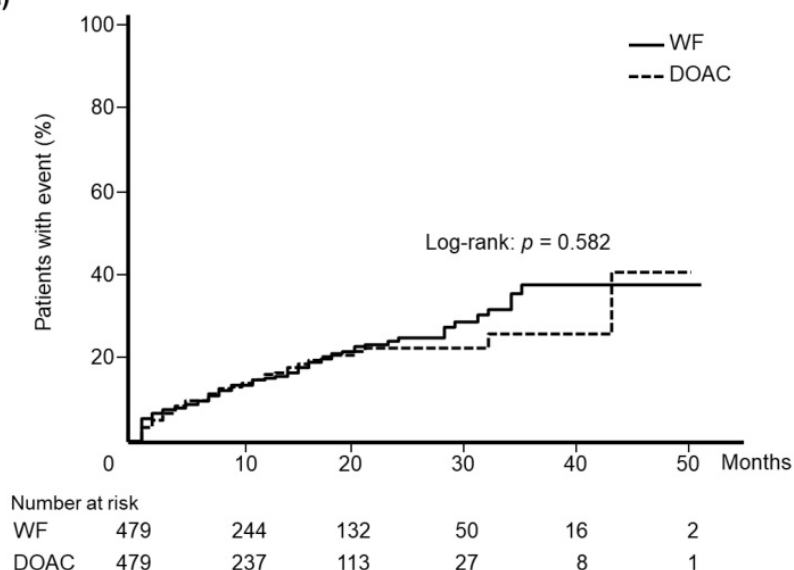

b)

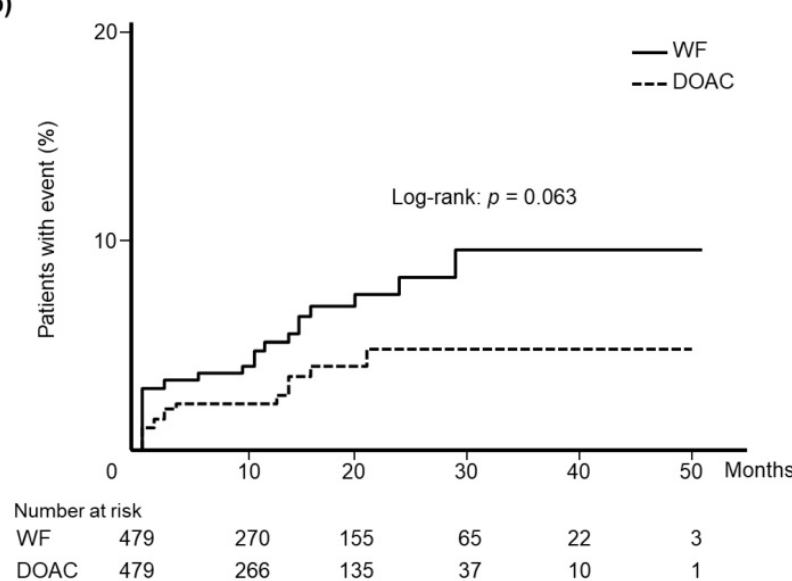

c)

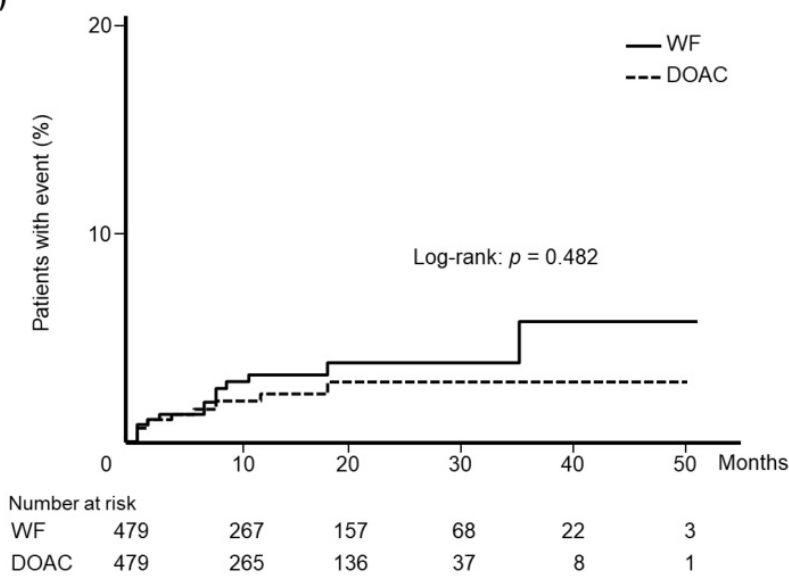

Fig. 4 Kaplan-Meier curves for the incidence of (a) total bleeding events, (b) gastrointestinal hemorrhage, and (c) intracranial hemorrhage in the propensity score matched cohorts. WF: warfarin, DOAC: direct oral anticoagulant.

\section{Discussion}

This study was designed to elucidate the difference in the risk of bleeding as a complication of anticoagulant therapy between WF and DOAC in patients with AF. A large claims database was used to evaluate the risk in the real-world clinical setting. Consequently, this study showed that there was no significant difference in the risk of total bleeding events between WF and DOAC in Japanese younger population with AF.

After the introduction of DOAC in 2011 in Japan, the claims database indicated that the prevalence of WF prescription decreased and that of DOAC increased gradually. A study using Japanese AF registry data supported this trend [10]. Globally, there has also been an increase in patients newly diagnosed with $\mathrm{AF}$, followed by increased use of DOAC and reduced use of WF $[14,15]$. In our study, according to patient characteristics, there was a difference in patient background factors between the WF and DOAC groups. The prevalence rates of concomitant use of antiplatelet agents, $\mathrm{H}_{2}$-receptor antagonists, and PPIs were significantly higher in the WF group compared to the DOAC group. Additionally, the prevalence rates of hypertension, renal dysfunction, and gastrointestinal dysfunction were also significantly higher in the WF group than the DOAC group. Furthermore, $\mathrm{CHADS}_{2}$ score was significantly higher in the WF group compared to the DOAC group. These results suggest that WF was predominantly used for patients with a high risk of stroke and relatively poor clinical condition. Due to limited clinical experience with DOAC therapy during the early stages of its introduction, DOACs might be more selectively used in patients with $\mathrm{AF}$ having a lower stroke risk.

Kaplan-Meier curves for total bleeding events and intracranial hemorrhage indicated that there were no significant differences in the incident rates between the WF and DOAC groups, but the incidence of gastrointestinal hemorrhage was significantly higher in the WF group than the DOAC group. Univariate analysis, not taking into account confounding factors, may lead to erroneous conclusions. Therefore, we conducted propensity score matching in order to reduce the effect of potential confounding. After matching, there were no significant differences in the incident rates of total bleeding events, gastrointestinal hemorrhage, and intracranial hemorrhage between the two groups. Similar results were reported in an observational study using registry data [10]. It is thus suggested that OAC status (WF or DOAC) does not influence the incidence of bleeding events.

Observational studies in general practice settings reported that some DOACs may have a higher risk of gastrointestinal hemorrhage than WF $[16,17]$. In Japan, after DOACs were introduced, major bleeding events increased in patients with a high risk of stroke and bleeding events in an observational, cohort study [18]. However, Cox proportional hazards regression model analysis showed that use of DOAC was not significantly different compared to the use of WF with 
regard to the incidence of bleeding events in our study. Many studies reported the efficacy and safety of DOAC compared with WF. Meta-analysis of these studies also reported that DOACs were similar to WF with regard to major bleeding events [5]. Even in clinical practice, other factors could be associated with bleeding events rather than WF or DOAC. In our study, factors such as age over 60 years, prior stroke or TIA and gastrointestinal dysfunction at baseline were predictors of bleeding events. Japanese patients with AF with a creatinine clearance $<30 \mathrm{ml} / \mathrm{min}$ showed a higher risk of major bleeding [19]; in contrast, renal dysfunction in our study did not have a statistically significant effect. Older age and prior stroke events have been reported as risk factors for bleeding [20]; our findings support these results.

This study has several limitations. First, the present data was derived from the JMDC claims database. The study population was selected from beneficiaries covered by the employees' health insurance system. Because most beneficiaries are working adults or their family members, the proportion of elderly patients aged $\geq 65$ years is low. Therefore, our study population was younger than those of other studies. Based on the characteristics of this database, the clinical background of the included patients may not be extrapolated to the general Japanese patient population. Second, there is the possibility that the ICD-10 code in the claims database does not accurately reflect clinical manifestations. The accuracy of physician diagnoses could not be verified, and the risk of misclassification cannot be ruled out. Third, there were many censored cases in the depicted Kaplan-Meier curve. Persistent use of WF or DOAC was influenced by older age [21] or anticoagulant therapy-naïve user status, respectively [22]. In patients with AF that are newly prescribed DOAC therapy, one in five patients changed to an alternate anticoagulant and one of every two patients did so within the first 6 months of therapy [23]. Fourth, appropriate prescription is important in pharmacotherapy; however, it was reported that more than half of DOAC prescriptions fell outside of recommended guidelines [24]. Apixaban and edoxaban have dose reduction criteria according to patients' body weight in Japan. However, body weight data was not collected because JMDC database did not contained it. Furthermore, we were able to collect information concerning the drug and prescription, but we had no data concerning actual intake by the patient. The potential influence of these factors on clinical outcomes is unknown. Fifth, we did not take account bleeding risk as a confounder. In patients with $\mathrm{AF}$ on anticoagulants, a validated specific bleeding risk score, such as PT-INR and HAS-BLED, should be used for assessment [25]. However, in this study using a claims database, information concerning PT-INR and HAS-BLED could not be obtained. Furthermore, we could not adjust for all confounders accounted for in this cohort study. There could still be residual confounding. It is necessary to pay attention when comparing the results between the present patient population and other cohorts with different indications.

\section{Conclusions}

The results of this historical cohort study using the JMDC claims database demonstrate that the bleeding risk of WF was comparable to that of DOAC in Japanese younger population. This study evaluated actual clinical data, including patients from various hospital sizes, hospital types, and regions. Analysis of database based on real world data, not registry database, revealing effectiveness and safety of DOAC and WF was of significance. There were some limitations. However, our results showed findings in agreement with those from RCTs. Harmonization of the result of RCTs and real-world data would be helpful for health care practitioners and would allow for improved clinical outcomes.

\section{Supplementary Material}

Supplementary figures and tables. http://www.medsci.org/v15p1686s1.pdf

\section{Acknowledgements}

Study concept and design: Mitsutaka Takada; Acquisition of data: Yuki Tanaka, Kazuki Nakakita; Analysis and interpretation of data: Yuki Tanaka, Satoshi Yokoyama, Mitsutaka Takada; Drafting of the manuscript: Satoshi Yokoyama, Kouichi Hosomi, Mitsutaka Takada; Revision of the manuscript: Mitsutaka Takada; Statistical analysis: Yuki Tanaka, Sotoshi Yokoyama, Mistukata Takada; Supervision: Mistutaka Takada.

\section{Competing Interests}

The authors have declared that no competing interest exists.

\section{References}

1. Patel MR, Mahaffey KW, Garg J, et al. Rivaroxaban versus warfarin in nonvalvular atrial fibrillation. N Engl J Med. 2011; 365: 883-91.

2. Giugliano RP, Ruff CT, Braunwald $E$, et al. Edoxaban versus warfarin in patients with atrial fibrillation. N Engl J Med. 2013; 369: 2093-104.

3. Connolly SJ, Ezekowitz MD, Yusuf S, et al. Dabigatran versus warfarin in patients with atrial fibrillation. N Engl J Med. 2009; 361: 1139-51.

4. Granger CB, Alexander JH, McMurray JJ, et al. Apixaban versus warfarin in patients with atrial fibrillation. N Engl J Med. 2011; 365: 981-92.

5. Ruff CT, Giugliano RP, Braunwald E, et al. Comparison of the efficacy and safety of new oral anticoagulants with warfarin in patients with atrial fibrillation: a meta-analysis of randomised trials. Lancet. 2014; 383: 955-62.

6. Camm AJ, Lip GY, De Caterina R, Savelieva I, et al. 2012 focused update of the ESC Guidelines for the management of atrial fibrillation: an update of the 2010 
ESC Guidelines for the management of atrial fibrillation. Developed with the special contribution of the European Heart Rhythm Association. Eur Heart J. 2012; 33: 2719-47.

7. January CT, Wann LS, Alpert JS, et al. 2014 AHA/ACC/HRS guideline for the management of patients with atrial fibrillation: a report of the American College of Cardiology/American Heart Association Task Force on practice guidelines and the Heart Rhythm Society. Circulation. 2014; 130: e199-267.

8. Spivey CA, Qiao Y, Liu X, et al. Discontinuation/Interruption of Warfarin Therapy in Patients with Nonvalvular Atrial Fibrillation. J Manag Care Spec Pharm. 2015; 21: 596-606.

9. Kachroo S, Hamilton M, Liu X, et al. Oral anticoagulant discontinuation in patients with nonvalvular atrial fibrillation. Am J Manag Care. 2013; 22: e1-8.

10. Yamashita Y, Uozumi R, Hamatani Y, et al. Current Status and Outcomes of Direct Oral Anticoagulant Use in Real-World Atrial Fibrillation Patients Fushimi AF Registry. Circ J. 2017; 81: 1278-85.

11. Kimura S, Sato T, Ikeda S, et al. Development of a database of health insurance claims: standardization of disease classifications and anonymous record linkage. J Epidemiol. 2010; 20: 413-9.

12. Gage BF, Waterman AD, Shannon W, et al. Validation of clinical classification schemes for predicting stroke: results from the National Registry of Atrial Fibrillation. JAMA. 2001; 285: 2864-70.

13. Rubin DB, Rosenbaum PR. The central role of the propensity score in observational studies for causal effects. Biometrika. 1983; 70: 41-55.

14. Camm AJ, Accetta G, Ambrosio G, et al. Evolving antithrombotic treatment patterns for patients with newly diagnosed atrial fibrillation. Heart. 2017; 103: 307-14.

15. Apenteng PN, Gao H, Hobbs FR, et al. Temporal trends in antithrombotic treatment of real-world UK patients with newly diagnosed atrial fibrillation: findings from the GARFIELD-AF registry. BMJ Open. 2018; 8: e018905.

16. Abraham NS, Singh S, Alexander GC, et al. Comparative risk of gastrointestinal bleeding with dabigatran, rivaroxaban, and warfarin: population based cohort study. BMJ. 2015; 350: h1857.

17. Chang HY, Zhou M, Tang W, et al. Risk of gastrointestinal bleeding associated with oral anticoagulants: population based retrospective cohort study. BMJ. 2015; 350: h1585.

18. Suzuki S, Otsuka T, Sagara K, et al. Nine-Year Trend of Anticoagulation Use, Thromboembolic Events, and Major Bleeding in Patients With Non-Valvular Atrial Fibrillation - Shinken Database Analysis. Circ J. 2016; 80: 639-49.

19. Abe M, Ogawa H, Ishii M, et al. Relation of Stroke and Major Bleeding to Creatinine Clearance in Patients Atrial Fibrillation (from the Fushimi AF Registry). Am J Cardiol. 2017; 119: 1229-37.

20. Potpara TS, Lip GY. Oral anticoagulant therapy in atrial fibrillation patients at high stroke and bleeding risk. Prog Cardiovasc Dis. 2015; 58: 177-94.

21. Wilke T, Groth A, Fuchs A, et al. Persistence with VKA treatment in newly treated atrial fibrillation patients: an analysis based on a large sample of 38,076 German patients. Eur J Clin Pharmacol. 2017; 73: 1437-47.

22. Manzoor BS, Lee TA, Sharp LK, et al. Real-World Adherence and Persistence with Direct Oral Anticoagulants in Adults with Atrial Fibrillation. Pharmacotherapy. 2017; 37: 1221-30.

23. Manzoor BS, Walton SM, Sharp LK, et al. High number of newly initiated direct oral anticoagulant users switch to alternate anticoagulant therapy. J Thromb Thrombolysis. 2017; 44: 435-41.

24. Diaz H, Bagheri H, Palmaro A, et al. Patterns of direct oral anticoagulant drug prescription in France in 2010-2013: a study in the Midi-Pyrénées area. Eur J Clin Pharmacol. 2018; 74: 945-51.

25. Roldán V, Marín F, Manzano-Fernández S, et al. The HAS-BLED score has better prediction accuracy for major bleeding than CHADS2 or CHA2DS2VASc scores in anticoagulated patients with atrial fibrillation. J Am Coll Cardiol. 2013; 62: 2199-204. 